\title{
CONSOLIDATIONS OF EXTENDED QI'S INEQUALITY AND BOUGOFFA'S INEQUALITY
}

\author{
Wenjun LiU, CHuncheng Li AND JiAnWEI DONG
}

Abstract. In this paper, an extension of Bougoffa's inequality is given, which gives a complete answer to an open problem posed by Bougoffa in [3]. Moreover, consolidations of extended Qi's inequality and Bougoffa's inequality are obtained.

Mathematics subject classification (2000): 26D15.

Key words and phrases: Qi's inequality, Bougoffa's inequality, integral inequality, Cauchy's mean value theorem, reversed Hölder inequality.

\section{REFERENCES}

[1] M. AKKouchI, On an integral inequality of Feng Qi, Divulgaciones Math., 13(1) (2005), 11-19.

[2] L. BougOFFA, Notes on Qi type integral inequalities, J. Inequal. Pure Appl. Math., 4(4) (2003), Art. 77.

[3] L. Bougoffa, An integral inequality similar to Qi's inequality, J. Inequal. Pure Appl. Math., 6(1) (2005), Art. 27.

[4] Y. Chen And J. Kimball, Note on an open problem of Feng Qi, J. Inequal. Pure Appl. Math., 7(1) (2006), Art. 4.

[5] J. C. KuAnG, Applied Inequalities, 3nd edition, Shandong Science and Technology Press, Jinan, China, 2004. (In Chinese)

[6] W. J. LiU, C. C. Li AND J. W. Dong, Note on Qi's inequality and Bougoffa's inequality, J. Inequal. Pure Appl. Math., 7(4) (2006), Art. 129.

[7] S. MAZOUZI AND F. QI, On an open problem regarding an integral inequality, J. Inequal. Pure Appl. Math., 4(2) (2003), Art. 31.

[8] T. K. Pogany, On an open problem of F. Qi, J. Inequal. Pure Appl. Math., 3(4) (2002), Art. 54.

[9] F. QI, Several integral inequalities, J. Inequal. Pure Appl. Math., 1(2) (2000), Art. 19.

[10] F. QI, A. J. LI, W. Z. ZHAO, D. W. NIU AND J. CAO, Extensions of Several integral inequalities, J. Inequal. Pure Appl. Math. ,7(3) (2006), Art. 107.

[11] K. W. YU AND F. QI, A short note on an integral inequality, RGMIA Res. Rep. Coll., 4(1) (2001), Art. $4,23-25$. 